\title{
Analysis of response rate with ANTI PD1/PD-L1 monoclonal antibodies in advanced solid tumors: a meta-analysis of randomized clinical trials
}

\author{
Alberto Carretero-González ${ }^{1}$, David Lora², Ismael Ghanem ${ }^{3}$, Jon Zugazagoitia ${ }^{1}$, \\ Daniel Castellano ${ }^{1}$, Juan M. Sepúlveda ${ }^{1}$, José A. López-Martin ${ }^{1}$, Luis Paz-Ares ${ }^{1}$ and \\ Guillermo de Velasco ${ }^{1}$ \\ ${ }^{1}$ Medical Oncology Service, University Hospital 12 de Octubre, Madrid, Spain \\ ${ }^{2}$ Clinical Research Unit (imas12-CIBERESP), University Hospital 12 de Octubre, Madrid, Spain \\ ${ }^{3}$ Medical Oncology Service, University Hospital La Paz, Madrid, Spain \\ Correspondence to: Guillermo de Velasco, email: gdvelasco.gdv@gmail.com \\ Keywords: AntiPD I/PDL 1; nivolumab; pembrolizumab; atezolizumab; response rate \\ Received: September 11,2017 Accepted: January 13,2018 Published: January 20, 2018 \\ Copyright: Carretero-González et al. This is an open-access article distributed under the terms of the Creative Commons Attribu- \\ tion License 3.0 (CC BY 3.0), which permits unrestricted use, distribution, and reproduction in any medium, provided the original \\ author and source are credited.
}

\section{ABSTRACT}

Background: Anti-PD1/PD-L1 monoclonal antibodies (mAbs) increase overall survival compared to standard of care (SOC) in different tumors. However, a proportion of patients (pts) will have progressive disease (PD) as best response. We conducted a meta-analysis to study the rates of response comparing these antibodies with SOC.

Methods: A search of published trials in MEDLINE and EMBASE analyzing antiPD1/PD-L1mAbs monotherapy compared to SOC. Relative risk (RR) with $95 \%$ confidence interval (CI) of response rates between groups was estimated. Subgroup analyses for location of primary tumor, number of previous treatment lines, selected population by PD-L1 expression and type of radiological assessment were made.

Results: Twelve studies accounting for 6,700 pts were included (anti-PD1/PDL1 mAbs: 3,451 pts; SOC: 3,249 pts [2,823 pts: chemotherapy, 426 pts: targeted therapy]). Adjusted response rates were (N, \%): Complete Response (CR) (69/3153, 2.19\%), Partial Response (PR) (596/3153, 18.90\%), Stable Disease (SD) (632/2463, $25.66 \%)$ and PD $(1027 / 2463,41.70 \%)$; and CR (16/2955, 0.54\%), PR (263/2955, $8.90 \%)$, SD $(835 / 2269,36.80 \%)$ and PD $(834 / 2269,36.76 \%)$ with anti-PD1/PD-L1 mAbs and SOC, respectively. Anti-PD1/PD-L1 mAbs improved CR rate (RR 3.48) and $P R$ rate ( $R R$ 2.27). There were no differences in the PD rate between groups ( $R R$ 1.10). Subgroup analyses showed an improvement in clinical benefit with anti-PD1/PD-L1 mAbs for melanoma (RR 1.59; 1.37-1.84 95\% CI) and those treated in the first line setting (RR 1.57; 1.27-1.95 95\% CI).

Conclusions: Anti-PD1/PD-L1 mAbs increase overall response rate compared to SOC without an increase in PD rate. Melanoma and pts treated in first line setting seem to have greater benefit with anti-PD1/PD-L1 mAbs.

Findings: In this systematic meta-analysis, anti-PD1/PD-L1 mAbs were associated with a greater overall response rate. Patients with melanoma and those managed in the first line setting seem to have an additional benefit with anti-PD1/ PD-L1 mAbs. 


\section{INTRODUCTION}

In recent years, immunotherapy has revolutionized the treatment of advanced cancer. Different methods have been shown to be effective in enhancing the host immune system in order to eradicate malignant cells. Immune checkpoint inhibitors targeting programmed cell death protein 1 (PD1) and programmed cell death-ligand protein 1(PD-L1) are the most encouraging field of research in this context [1-4].

PD1 is a cell surface protein expressed on $\mathrm{T}$ lymphocytes that binds its ligands (PD-L1 and Programmed cell death-ligand protein 2 [PD-L2]), present on the cell membrane of several types of cells, including tumor cells.

The PD1/PD-L1 axis represents a native co-inhibitory pathway that produces a negative effect on immune response activation by inducing a state of anergy in T lymphocytes [4]. Specifically, the PD1/PD-L1 pathway serves as a mechanism of peripheral immune tolerance by which tumor cells can evade the immune response and develop and become metastatic. This signaling system might also have a bearing on the priming of $\mathrm{T}$ lymphocytes in lymph nodes with a negative impact $[1,4]$. PD-L1 expression in tumor cells is associated with poor prognosis in several tumor types [5-7].

The importance of this pathway has been demonstrated with the development of several monoclonal antibodies (mAbs) targeting PD1 (nivolumab, pembrolizumab) or PD-L1 (atezolizumab, durvalumab, avelumab). These drugs have shown exceptional results in many tumor types, with improved overall survival and a benefit in the overall response rate (with potentially more marked benefits in selected groups such as those with higher PD-L1 expression) [8-12]. However, the benefit in terms of progression-free survival and the progressive disease (PD) rate has been less certain.

Considering that Response Evaluation Criteria in Solid Tumors version 1.1 (RECIST v1.1) criteria may not be the most appropriate tool to evaluate the anti-cancer effect of immunotherapy, they are the common assessment in most of the clinical trials evaluating anti-PD1/PD-L1 antibodies [13, 14]. Either PD or clinical benefit rate may constitute a relevant efficacy endpoint, and some trials would have reported negative outcomes for that specific endpoint (such as CheckMate 025, CheckMate 057 or CheckMate 141) $[8,15,16]$.

In order to understand the overall benefit in terms of response rates with novel anti-PD1/PD-L1 mAbs, we performed a meta-analysis of published phase II/III randomized clinical trials (RCTs) to assess the type of response achieved with these mAbs in monotherapy, and compared to the standard of care (SOC).

\section{PATIENTS AND METHODS}

\section{Literature search and inclusion criteria}

We identified all RCTs that compared anti-PD1/ PD-L1 mAbs in monotherapy with a non-immunotherapy control arm; this included Nivolumab $\left(\mathrm{Opdivo}^{\circledR}\right)$, Pembrolizumab (Keytruda ${ }^{\circledR}$ ), Atezolizumab (Tecentric ${ }^{\circledR}$ ), Durvalumab (MEDI4736) or Avelumab (MSB0010718C). An independent search of published studies from 1st January 2000 to 15th May 2017 in MEDLINE and EMBASE was performed. The time period was chosen commencing in 2000 because of the standardization of the clinical trial response rate with the publication of RECIST criteria. The following search terms were used: "nivolumab", "pembrolizumab", "atezolizumab", "durvalumab" and "avelumab". The review was restricted to RCTs in human subjects published in English. Abstract proceedings and virtual meeting presentations containing the same terms from the American Society of Clinical Oncology and the European Society of Medical Oncology conferences held between January 2010 and 15th May 2017 were also used to identify relevant clinical trials. We reviewed each publication, and only the most recent or complete report of RCTs was included when duplicate publications were identified. On 15th May 2017, the online updated manufacturers' package inserts of nivolumab, pembrolizumab, atezolizumab, durvalumab, and avelumab were also reviewed to identify relevant information not previously reported in published clinical trials. No placebocontrolled randomized trials including these agents were found. Trials involving anti-PD1/PD-L1 mAbs in combination with other agents (either immunotherapeutic, chemotherapeutic or targeted therapies) were excluded in order to observe clinical responses to anti-PD1/PDL1 mAbs in monotherapy alone. Selected response rates included those proposed by RECIST v1.1 criteria: complete response $(\mathrm{CR})$ rate, partial response $(\mathrm{PR})$ rate, stable disease (SD) rate and progressive disease (PD) rate. Trials that met the following criteria were included in the meta-analysis: randomized phase II and III trials, prospective clinical trials in patients with cancer, and trials with response rate data available. Two reviewers (A. C-G. and G.d.V.) independently evaluated studies for eligibility.

\section{Data extraction and clinical end points}

Data was extracted as already outlined, using a preliminary screen of two investigators (A. C-G. and G. d. V.) according to Quality of Reporting of Meta-Analyses (QUORUM) guidelines. Variables collected and included were: first author's surname, year of publication, National Clinical Trials (NCT) registry number, type of underlying malignancy, number of previous treatments received, selection of population by PD-L1 expression on tumor cells (yes/no), type of radiological assessment of response (by investigator or by independent central review), phase of the trial, number of enrolled subjects, number of patients included in the overall response rate (ORR; considered as the sum of $\mathrm{CR}$ and $\mathrm{PR}$ ) analysis, treatment arms, number of patients in the anti-PD1/PD-L1 mAbs and control groups, name of the anti-PD1/PD-L1 mAb (nivolumab, pembrolizumab, atezolizumab, durvalumab, 
avelumab), median age and response rates ( $\% \mathrm{CR}, \mathrm{PR}, \mathrm{SD}$ and PD) obtained per treatment group.

\section{Statistical analysis}

All statistical analyses were performed using meta package $[17,18]$. For binary outcome, CR/PR/SD/PD risk ratios with CIs were used as the measure of effect of the anti-PD1/PD-L1 mAbs arm versus the SOC (either chemotherapy or targeted therapy) arm (control arm). Statistical heterogeneity among trials included in the metaanalysis was assessed using I2 statistics, which estimates the percentage of total variation across studies due to heterogeneity rather than chance [19]. The assumption of homogeneity was considered invalid for $p$-value $<0.05$. We pooled studies using random and fixed-effects models depending on the heterogeneity of the studies included. When substantial heterogeneity was not observed, the summary estimate calculated on the basis of the fixedeffects model was reported using the Mantel-Haenszel method; otherwise, the random-effects model was reported by using the DerSimonian and Laird method that considers both within-study and between-study variations [20].Subgroup analyses were conducted by underlying malignancy, number of previous treatments received, type of radiological assessment of response (by investigator or by independent central review) or selection of population by PD-L1 expression on tumor cells (yes/no). In addition, publication bias was evaluated through funnel plots (i.e., plots of study results against precision).

\section{RESULTS}

\section{Study selection}

The flow chart shows the studies selected (Figure 1); 454 studies were reviewed through our selection process for RCTs. Exclusions were: (i) letters, editorials, reviews and retrospective studies (375 studies); (ii) expandedaccess studies with no control arm and early-phase I/II or non-RCTs (54 studies); and (iii) studies with no adequate control arm (13 studies). Twelve trials met the criteria for inclusion in the meta-analysis (randomized phase II/III trials with anti-PD1/PD-L1 monotherapy and a control arm that did not contain immunotherapeutic agents).

Baseline characteristics of each trial are presented in Table 1 [8-10, 15, 16, 21-27]. Six trials were performed in patients with non-small cell lung cancer, three in melanoma, one in renal cell carcinoma, one in squamous cell cancer of the head and neck, and one in urothelial carcinoma. Two studies were performed in the first-line setting [23, 25], while the remainder assessed subsequent treatment lines. All studies had two treatment arms but two (both with three arms). Two studies had selected populations according to PD-L1 expression on tumor cells (one with at least $1 \%$ of expressing tumor cells and the other with at least $50 \%$ ). A total of 6938 patients were available for the meta-analysis, specifically 6700 patients with measurable disease: 3451 patients were assigned to anti-PD1/PDL1 mAbs arms (nivolumab 1407, pembrolizumab 1475, atezolizumab 569, durvalumab 0, avelumab 0), and 3249 were assigned to SOC arms (chemotherapy 2823, targeted therapy 426 [including everolimus 411 and cetuximab 15]). Three of these studies presented incomplete information on response rates (without distinguishing among CR, PR or SD rates); the response proportions were reviewed to address this issue $[10,24,25]$. Only solid tumors were included. ORR was a co-primary end point for efficacy in only one of these studies, in addition to overall survival [22]; it was evaluated by RECIST v1.1 criteria in all of them. Five studies were centrally assessed $[9,22,24$, $25,27]$, while seven were assessed by the investigator $[8,10,15,16,21,23,26]$. All RCTs were sponsored by pharmaceutical companies.

\section{Incidence and relative risk of $\mathrm{CR}, \mathrm{PR}$ and $\mathrm{SD}$ rates}

In patients who received anti-PD1/PD-L1 mAbs, CR was obtained in 69/3153 patients $(2.19 \%)$ and $\mathrm{PR}$ was obtained in 596/3153 (18.90\%). Compared to patients in a non-immunotherapy control arm, those treated with an anti-PD1/PD-L1 mAb were more likely to have CR (RR 3.48; 95\% CI 2.11-5.76, $p<0.0001$ ) and PR (RR 2.27 ; 95\% CI 1.67-3.09, $p<0.0001$ ) (Figure 2A and 2B). On the other hand, patients treated with immunotherapy achieved a response classified as SD in 632/2463 patients $(25.66 \%)$, while this result was observed in $835 / 2269$ $(36.80 \%)$ of those who received a non-immunotherapy treatment; this type of response was more frequently achieved in patients managed with chemotherapy or targeted therapy, compared to anti-PD1/PD-L1 mAbs (RR $0.71 ; 95 \%$ CI $0.62-0.81, p<0.0001$ ) (Figure 2 C).

\section{Incidence and relative risk of $P D$ and clinical benefit rates}

Our analysis found no statistically significant difference between the two treatment groups in responses classified as PD (RR 1.10; 95\% CI 0.89-1.37, p 0.37); specifically, PD was obtained in $1027 / 2463$ patients $(41.70 \%)$ and $834 / 2269$ patients $(36.76 \%)$ treated with anti-PD1/PD-L1 mAbs and control arm group, respectively (Figure 2D). Therefore, the clinical benefit rate (considered as the sum of CR, PR and SD) is similar between both groups (RR 1.11; 95\% CI 0.95-1.31, p 0.09), with estimated proportions of $1171 / 2463$ patients (47.54\%) in the immunotherapy arm group, and 1050/2269 $(46.28 \%)$ in the control arm group.

\section{Subgroup analyses}

Based on the primary tumor analysis, melanoma patients treated with anti-PD1/PD-L1 mAbs tended to have 
a higher SD rate compared to other locations (RR 0.83; 95\% CI $0.67-1.04$ for melanoma vs RR $0.75 ; 95 \%$ CI 0.60-0.95 for lung cancer vs RR 0.61; 95\% CI 0.53-0.69 for other tumors; data not shown) with no differences in objective response rates; melanoma patients treated with anti-PD1/PD-L1 mAbs exhibited a lower PD rate (RR
0.76; 95\% CI 0.67-0.85 for melanoma vs RR 1.47; 95\% CI 1.26-1.71 for lung cancer vs RR 1.32; 95 CI 1.16-1.51 for other tumors; data not shown) and a better clinical benefit rate (RR 1.59; 95\% CI 1.37-1.84 for melanoma vs RR 0.91; 95\% CI 0.78-1.06 for lung cancer vs RR 0.94; 95\% CI 0.86-1.03 for other tumors; Figure 3A).

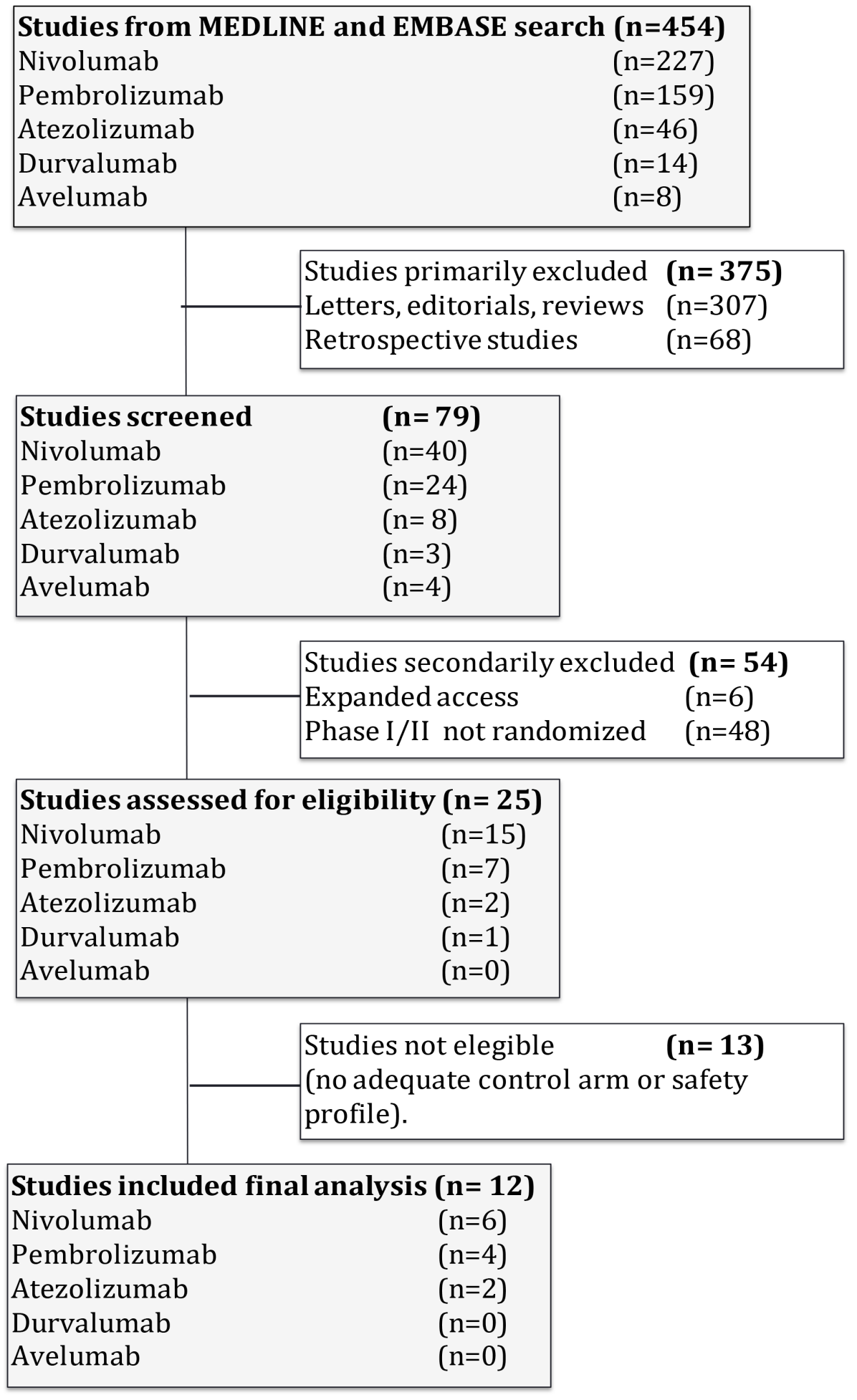

Figure 1: Flow diagram of the systematic review. 
Table 1: List of clinical trials included in the meta-analysis

\begin{tabular}{|c|c|c|c|c|c|c|}
\hline & Reference & $\mathbf{P h}$ & Masking & Histology & No. & Treatment arms \\
\hline \multicolumn{7}{|c|}{ Nivolumab } \\
\hline \multirow[t]{2}{*}{1} & Brahmer J. et al. (2015) & 3 & Open-label & $\operatorname{NSCLC~(Sq)~}$ & 272 & Nivolumab \\
\hline & & & & & & Docetaxel \\
\hline \multirow[t]{2}{*}{2} & Borghaei H. et al. (2015) & 3 & Open-label & NSCLC (Non-Sq) & 582 & Nivolumab \\
\hline & & & & & & Docetaxel \\
\hline \multirow[t]{2}{*}{3} & Motzer RJ. et al. (2015) & 3 & Open-label & Renal & 821 & Nivolumab \\
\hline & & & & & & Everolimus \\
\hline \multirow[t]{2}{*}{4} & Ferris RL. et al. (2016) & 3 & Open-label & Head\&Neck & 361 & Nivolumab \\
\hline & & & & & & MTX/Docetaxel/Cetuximab \\
\hline \multirow[t]{2}{*}{5} & Robert C. et al. (2015) & 3 & Double-blind & Melanoma & 418 & Nivolumab \\
\hline & & & & & & Dacarbazine \\
\hline \multirow[t]{2}{*}{6} & Weber JS. et al. (2015) & 3 & Open-label & Melanoma & 405 & Nivolumab \\
\hline & & & & & & Chemotherapy \\
\hline \multicolumn{7}{|c|}{ Pembrolizumab } \\
\hline \multirow[t]{3}{*}{7} & Herbst RS. et al. (2016) & $2 / 3$ & Open-label & NSCLC (PDL1 > 1\%) & 1034 & Pembrolizumab $2 \mathrm{mg} / \mathrm{Kg}$ \\
\hline & & & & & & Pembrolizumab $10 \mathrm{mg} / \mathrm{kg}$ \\
\hline & & & & & & Docetaxel \\
\hline \multirow[t]{3}{*}{8} & Ribas A. et al. (2015) & 2 & Open-label & Melanoma & 540 & Pembrolizumab 2 mg/kg \\
\hline & & & & & & Pembrolizumab $10 \mathrm{mg} / \mathrm{kg}$ \\
\hline & & & & & & Chemotherapy \\
\hline \multirow[t]{2}{*}{9} & Reck M. et al. (2016) & 3 & Open-label & NSCLC (PDL1 > 50\%) & 305 & Pembrolizumab \\
\hline & & & & & & Chemotherapy \\
\hline \multirow[t]{2}{*}{10} & Bellmunt J. et al. (2017) & 3 & Open-label & Urothelial carcinoma & 542 & Pembrolizumab \\
\hline & & & & & & $\begin{array}{c}\text { Docetaxel/Paclitaxel/ } \\
\text { Vinflunine }\end{array}$ \\
\hline \multicolumn{7}{|c|}{ Atezolizumab } \\
\hline \multirow[t]{2}{*}{11} & Fehrenbacher L. et al. (2016) & 2 & Open-label & NSCLC & 287 & Atezolizumab \\
\hline & & & & & & Docetaxel \\
\hline \multirow[t]{2}{*}{12} & Rittmeyer A. et al. (2016) & 3 & Open-label & NSCLC & 850 & Atezolizumab \\
\hline & & & & & & Docetaxel \\
\hline
\end{tabular}

Based on line of therapy, anti-PD1/PD-L1 mAbs as first-line therapy achieved a lower PD rate compared to subsequent lines of therapy (RR 0.68; 95\% CI 0.53-0.86 for first line vs RR 1.17; 95\% CI 0.94-1.44 for subsequent lines; data not shown) and, therefore, a better clinical benefit rate (RR 1.57; 95\% CI 1.27-1.95 for first-line vs RR 1.06; 95\% CI 0.91-1.23 for subsequent lines; Figure 3B).

No differences were seen in the analyses based on PD-L1 expression on tumor cells or by type of radiological assessment (central vs investigator).

\section{DISCUSSION}

This comprehensive analysis including more than 6000 patients has shown that anti-PD1/PD-L1 mAbs are associated with an improved response rate compared to SOC in different solid tumors, with no differences in the rate of progression as best response. Overall, immune checkpoint blockade (ICB, including anti-PD1/PD-L1 $\mathrm{mAbs}$ ) offers a similar clinical benefit rate with increased ORR. Additionally, studies on anti-PD1/PD-L1 mAbs have shown that patients may achieve durable responses (data not shown). Of note, it has been recently shown that PFS of patients who obtain CR is superior than those who achieve PR; as we have shown in this meta-analysis both type of responses are increased with anti-PD1/PDL1 mAbs independently [28]. Thus, from this perspective, anti-PD1/PD-L1 mAbs also represent a better strategy for tumor shrinkage compared to chemotherapy or targeted therapy in most cases. 
Notably, melanoma patients could present a greater benefit compared to other locations, with improved clinical benefit rate.

Different studies have attempted to determine whether the line of therapy with ICB should be used before or after SOC. In our study, patients achieved better outcomes at first-line, although these data should be taken with caution as many corresponded to melanoma patients. PD-L1 expression has been the most widely studied biomarker for ICB in recent years [24, 25]. The subanalysis based on PD-L1 expression was limited by the small number of events in each subgroup, so no conclusions could be drawn and further studies are warranted. In addition, the type of radiological review (centrally or by investigator) had no effect on these results, despite the general recommendation of a response assessment by an independent central committee.

ICB has been successfully developed in many solid tumors and hematologic malignancies because of its improvement in overall survival and reduction in toxicities compared to SOC. ORR has been considered an important parameter to consider in the development of antineoplastic drugs. It is one of the main efficacy endpoints in early phase clinical trials (phase II), and also acts as a surrogate marker for survival in phase III trials [29]. ICB has also been shown to increase overall response rate (CR and $\mathrm{PR}$ ), but it was unclear until now whether the PD rate (in other words, clinical benefit rate) also improved, since many patients obtain $\mathrm{PD}$ as best response in clinical practice with these agents. A new pattern of progression has also been described in patients treated with anti-PD1/PD-L1 mAbs, termed as hyperprogressive disease (defined as a RECIST progression at first evaluation and as a $\geq$ two-fold increase in the tumor growth rate), and is associated with poorer survival. This phenomenon was present up to $9 \%$ of the patients treated with ICB, and may increase with age (up to $19 \%$ in elderly patients) [30]. This type of phenomenon suggests the need for predictive biomarkers that could be extremely relevant in some situations where tumor shrinkage may be critical (for instance, symptomatic patients with high tumor burden).

For many years, different response criteria have been published in order to standardize results of new

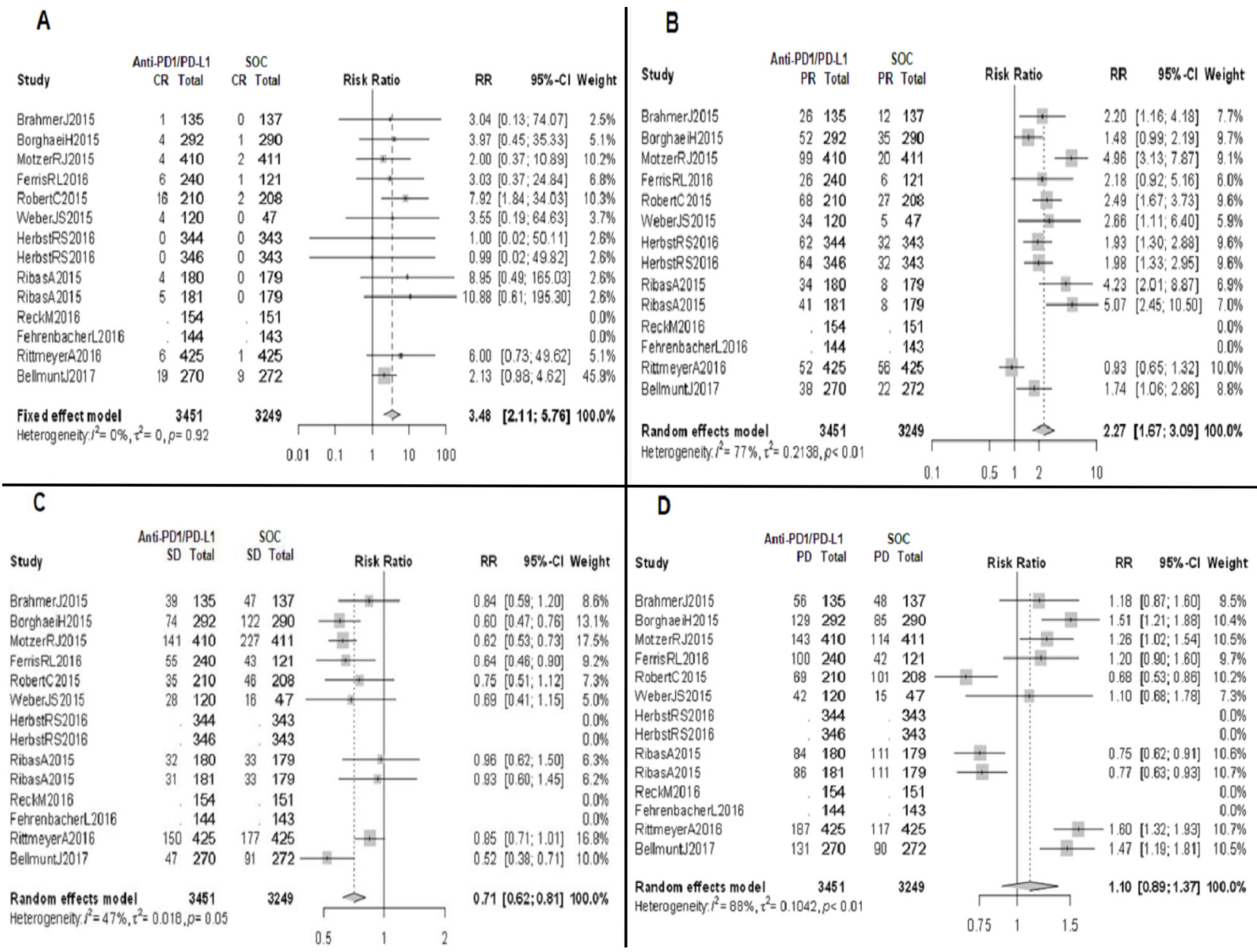

Figure 2: Forrest plot diagrams: Relative risk (RR) with 95\% confidence interval (CI) of responses between anti-PD1/ PD-L1 mAbs and standard of care (SOC). (A) Complete response (CR). (B) Partial response (PR). (C) Stable disease (SD). (D) Progressive disease (PD). 
developing drugs [29, 31-33]. However, due to the specific mechanism of action of immunotherapy (which differs from that of chemotherapy in a potential initial tumor swelling followed by a reduction of tumor cells termed pseudoprogression - rather than direct killing of malignant cells), the most established criteria (RECIST v1.1) may not be the best tool to evaluate the anti-cancer effect of immune checkpoint inhibitors [13, 14, 34, 35]. This represents another limitation in analyzing the objective response, since $3-4 \%$ of patients progressing to ICB in the first scan may still benefit from treatment beyond progression. These figures could increase up to $10 \%$ in melanoma $[13,35]$.

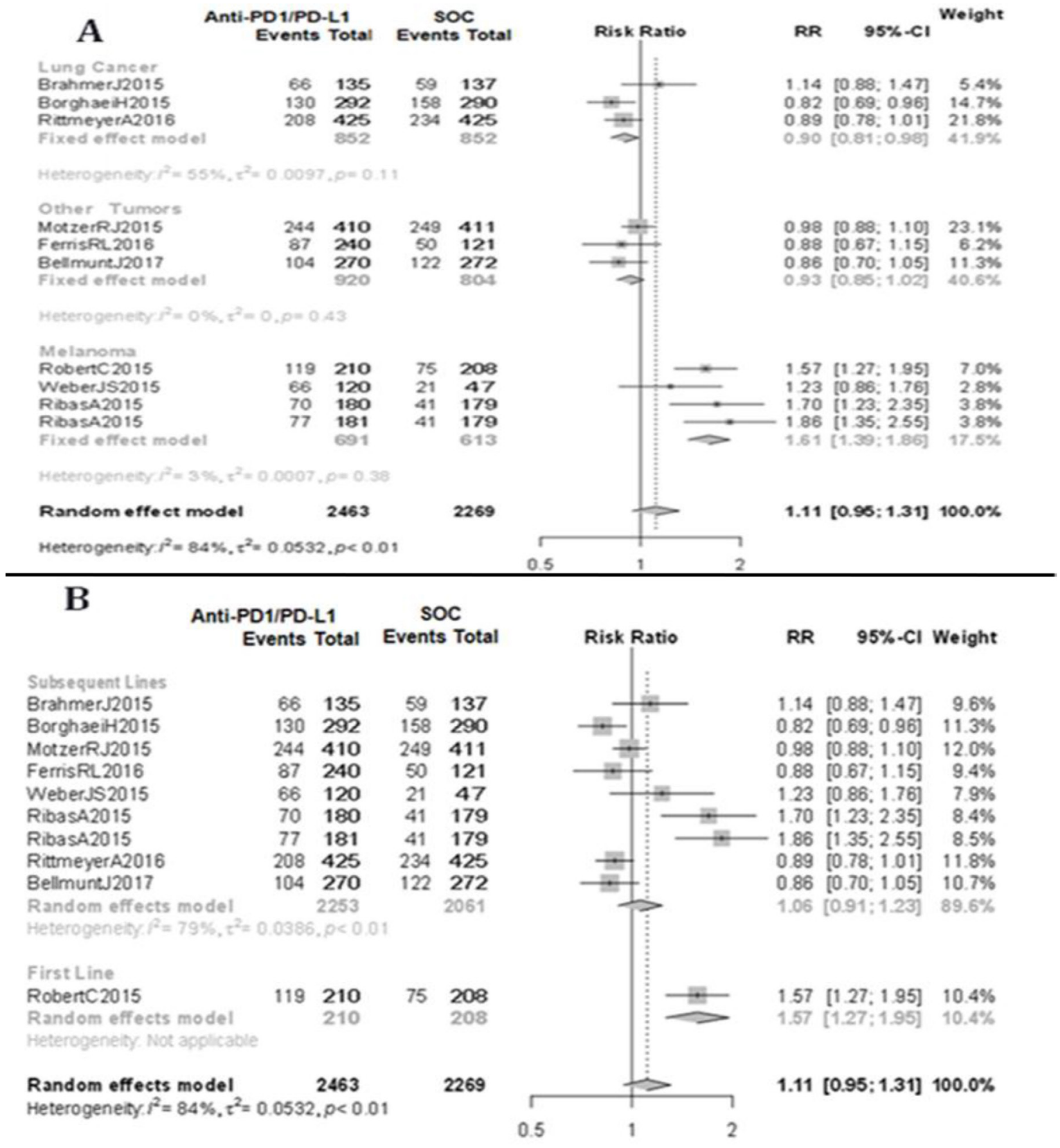

Figure 3: Clinical benefit. (A) Subgroup analysis by type of underlying malignancy. (B) Subgroup analysis by number of previous treatments received. Standard of care (SOC). Relative risk (RR). Confidence interval (CI). 
radiological assessment, selected population in some studies and perhaps other concomitant conditions not recorded), so we cannot draw overall conclusions, although we can obtain a general overview. In addition, we were unable to retrieve patient level data, although some studies have suggested trial-level and patient-level meta-analyses may reach comparable outcomes [39]. In this study, we did not evaluate therapy combinations (chemotherapy with immunotherapy or more than one immunotherapeutic agent), which have already been shown to achieve a greater response rate compared to monotherapy, but with an apparent concomitant increase in toxicities [40]. We could not compare anti-PD1 mAbs vs anti-PD-L1 mAbs because of the paucity of results with the latter. Finally, we have not taken into account ORRrelated parameters, such as time to response or duration of response; these data would have improved the evaluation of the real benefit with immunotherapy compared to SOC.

In conclusion, in this meta-analysis, anti-PD1/PD-L1 $\mathrm{mAbs}$ were found to increase the overall response rate compared to $\mathrm{SOC}$, with no increase in the $\mathrm{PD}$ rate according to RECIST v1.1 criteria. Patients with melanoma and those treated in the first-line setting seem to obtain greater benefit with anti-PD1/PD-L1 mAbs.

\section{CONFLICTS OF INTEREST}

The authors declare no conflicts of interest.

\section{FUNDING}

This research did not receive any specific grant from funding agencies in the public, commercial, or not-forprofit sectors.

\section{REFERENCES}

1. Chen DS, Mellman I. Oncology meets immunology: the cancer-immunity cycle. Immunity. 2013; 39:1-10. https:// doi.org/10.1016/j.immuni.2013.07.012.

2. Mandal R, Chan TA. Personalized Oncology Meets Immunology: The Path toward Precision Immunotherapy. Cancer Discov. 2016; 6:703-13. https://doi.org/10.1158/21598290.CD-16-0146.

3. Palucka AK, Coussens LM. The Basis of Oncoimmunology. Cell. 2016; 164:1233-47. https://doi.org/10.1016/j. cell.2016.01.049.

4. Boussiotis VA. Molecular and Biochemical Aspects of the PD-1 Checkpoint Pathway. N Engl J Med. 2016; 375: 1767-78. https://doi.org/10.1056/NEJMra1514296.

5. Yu J, Wang X, Teng F, Kong L. PD-L1 expression in human cancers and its association with clinical outcomes. OncoTargets Ther. 2016; 9:5023-39. https://doi. org/10.2147/OTT.S105862.
6. Zhang Y, Kang S, Shen J, He J, Jiang L, Wang W, Guo Z, Peng G, Chen G, He J, Liang W. Prognostic Significance of Programmed Cell Death 1 (PD-1) or PD-1 Ligand 1 (PD-L1) Expression in Epithelial-Originated Cancer: A MetaAnalysis. Medicine (Baltimore). 2015; 94:e515. https://doi. org/10.1097/MD.0000000000000515.

7. Tamura T, Ohira M, Tanaka H, Muguruma K, Toyokawa T, Kubo N, Sakurai K, Amano R, Kimura K, Shibutani M, Maeda K, Hirakawa K. Programmed Death-1 Ligand-1 (PDL1) Expression Is Associated with the Prognosis of Patients with Stage II/III Gastric Cancer. Anticancer Res. 2015; 35:5369-76.

8. Borghaei H, Paz-Ares L, Horn L, Spigel DR, Steins M, Ready NE, Chow LQ, Vokes EE, Felip E, Holgado E, Barlesi F, Kohlhäufl M, Arrieta O, et al. Nivolumab versus Docetaxel in Advanced Nonsquamous Non-Small-Cell Lung Cancer. N Engl J Med. 2015; 373:1627-39. https:// doi.org/10.1056/NEJMoa1507643.

9. Ribas A, Puzanov I, Dummer R, Schadendorf D, Hamid O, Robert C, Hodi FS, Schachter J, Pavlick AC, Lewis KD, Cranmer LD, Blank CU, O’Day SJ, et al. Pembrolizumab versus investigator-choice chemotherapy for ipilimumabrefractory melanoma (KEYNOTE-002): a randomised, controlled, phase 2 trial. Lancet Oncol. 2015; 16:908-18. https://doi.org/10.1016/S1470-2045(15)00083-2.

10. Fehrenbacher L, Spira A, Ballinger M, Kowanetz M, Vansteenkiste J, Mazieres J, Park K, Smith D, ArtalCortes A, Lewanski C, Braiteh F, Waterkamp D, He P, et al. Atezolizumab versus docetaxel for patients with previously treated non-small-cell lung cancer (POPLAR): a multicentre, open-label, phase 2 randomised controlled trial. Lancet Lond Engl. 2016; 387:1837-46. https://doi. org/10.1016/S0140-6736(16)00587-0.

11. Antonia S, Goldberg SB, Balmanoukian A, Chaft JE, Sanborn RE, Gupta A, Narwal R, Steele K, Gu Y, Karakunnel JJ, Rizvi NA. Safety and antitumour activity of durvalumab plus tremelimumab in non-small cell lung cancer: a multicentre, phase 1b study. Lancet Oncol. 2016; 17: 299-308. https://doi.org/10.1016/S1470-2045(15)00544-6.

12. Kaufman HL, Russell J, Hamid O, Bhatia S, Terheyden P, D'Angelo SP, Shih KC, Lebbé C, Linette GP, Milella M, Brownell I, Lewis KD, Lorch JH, et al. Avelumab in patients with chemotherapy-refractory metastatic Merkel cell carcinoma: a multicentre, single-group, open-label, phase 2 trial. Lancet Oncol. 2016; 17:1374-85. https://doi. org/10.1016/S1470-2045(16)30364-3.

13. Wolchok JD, Hoos A, O’Day S, Weber JS, Hamid O, Lebbe C, Maio M, Binder M, Bohnsack O, Nichol G, Humphrey R, Hodi FS. Guidelines for the Evaluation of Immune Therapy Activity in Solid Tumors: Immune-Related Response Criteria. Clin Cancer Res. 2009; 15:7412-20. https://doi. org/10.1158/1078-0432.CCR-09-1624.

14. Hodi FS, Hwu WJ, Kefford R, Weber JS, Daud A, Hamid O, Patnaik A, Ribas A, Robert C, Gangadhar TC, Joshua AM, Hersey P, Dronca R, et al. Evaluation of Immune-Related 
Response Criteria and RECIST v1.1 in Patients With Advanced Melanoma Treated With Pembrolizumab. J Clin Oncol. 2016; 34:1510-7. https://doi.org/10.1200/ JCO.2015.64.0391

15. Motzer RJ, Escudier B, McDermott DF, George S, Hammers HJ, Srinivas S, Tykodi SS, Sosman JA, Procopio G, Plimack ER, Castellano D, Choueiri TK, Gurney H, et al. Nivolumab versus Everolimus in Advanced Renal-Cell Carcinoma. N Engl J Med. 2015; 373:1803-13. https://doi. org/10.1056/NEJMoa1510665.

16. Ferris RL, Blumenschein G, Fayette J, Guigay J, Colevas AD, Licitra L, Harrington K, Kasper S, Vokes EE, Even C, Worden F, Saba NF, Iglesias Docampo LC, et al. Nivolumab for Recurrent Squamous-Cell Carcinoma of the Head and Neck. N Engl J Med. 2016; 375:1856-67. https://doi. org/10.1056/NEJMoa1602252.

17. Schwarzer G. Meta: An R package for meta-analysis. R News. 2007; 7:40-5.

18. R Core Team. R: A language and environment for statistical computing. R Foundation for Statistical Computing. Vienna, Austria; 2016. Available from https://www.R-project.org/.

19. Higgins JP, Thompson SG, Deeks JJ, Altman DG. Measuring inconsistency in meta-analyses. BMJ. 2003; 327:557-60. https://doi.org/10.1136/bmj.327.7414.557.

20. DerSimonian R, Laird N. Meta-analysis in clinical trials. Control Clin Trials. 1986; 7:177-88.

21. Brahmer J, Reckamp KL, Baas P, Crinò L, Eberhardt WE, Poddubskaya E, Antonia S, Pluzanski A, Vokes EE, Holgado E, Waterhouse D, Ready N, Gainor J, et al. Nivolumab versus Docetaxel in Advanced SquamousCell Non-Small-Cell Lung Cancer. N Engl J Med. 2015; 373:123-35. https://doi.org/10.1056/NEJMoa1504627.

22. Weber JS, D'Angelo SP, Minor D, Hodi FS, Gutzmer R, Neyns B, Hoeller C, Khushalani NI, Miller WH, Lao CD, Linette GP, Thomas L, Lorigan P, et al. Nivolumab versus chemotherapy in patients with advanced melanoma who progressed after anti-CTLA-4 treatment (CheckMate 037): a randomised, controlled, open-label, phase 3 trial. Lancet Oncol. 2015; 16:375-84. https://doi.org/10.1016/ S1470-2045(15)70076-8.

23. Robert C, Long GV, Brady B, Dutriaux C, Maio M, Mortier L, Hassel JC, Rutkowski P, McNeil C, Kalinka-Warzocha E, Savage KJ, Hernberg MM, Lebbé C, et al. Nivolumab in previously untreated melanoma without BRAF mutation. N Engl J Med. 2015; 372:320-30. https://doi.org/10.1056/ NEJMoa1412082.

24. Herbst RS, Baas P, Kim DW, Felip E, Pérez-Gracia JL, Han JY, Molina J, Kim JH, Arvis CD, Ahn MJ, Majem M, Fidler MJ, de Castro G, et al. Pembrolizumab versus docetaxel for previously treated, PD-L1-positive, advanced non-smallcell lung cancer (KEYNOTE-010): a randomised controlled trial. Lancet Lond Engl. 2016; 387:1540-50. https:/doi. org/10.1016/S0140-6736(15)01281-7.
25. Reck M, Rodríguez-Abreu D, Robinson AG, Hui R, Csőszi T, Fülöp A, Gottfried M, Peled N, Tafreshi A, Cuffe S, O'Brien M, Rao S, Hotta K, et al. Pembrolizumab versus Chemotherapy for PD-L1-Positive Non-Small-Cell Lung Cancer. N Engl J Med. 2016; 375:1823-33. https://doi. org/10.1056/NEJMoa1606774.

26. Rittmeyer A, Barlesi F, Waterkamp D, Park K, Ciardiello F, von Pawel J, Gadgeel SM, Hida T, Kowalski DM, Dols MC, Cortinovis DL, Leach J, Polikoff J, et al. Atezolizumab versus docetaxel in patients with previously treated non-small-cell lung cancer (OAK): a phase 3, openlabel, multicentre randomised controlled trial. Lancet Lond Engl. 2017; 389:255-65. https://doi.org/10.1016/ S0140-6736(16)32517-X.

27. Bellmunt J, de Wit R, Vaughn DJ, Fradet Y, Lee JL, Fong L, Vogelzang NJ, Climent MA, Petrylak DP, Choueiri TK, Necchi A, Gerritsen W, Gurney H, et al. Pembrolizumab as Second-Line Therapy for Advanced Urothelial Carcinoma. N Engl J Med. 2017; 376:1015-26. https://doi.org/10.1056/ NEJMoa1613683.

28. Robert C, Long GV, Schachter J, Arance A, Grob JJ, Mortier L. Long-term outcomes in patients (pts) with ipilimumab (ipi)naive advanced melanoma in the phase 3 KEYNOTE-006 study who completed pembrolizumab (pembro) treatment. Journal of Clinical Oncology 2017; 35:9504-9504. https:// doi.org/10.1200/JCO.2017.35.15_suppl.9504.

29. Therasse P, Arbuck SG, Eisenhauer EA, Wanders J, Kaplan RS, Rubinstein L, Verweij J, Van Glabbeke M, van Oosterom AT, Christian MC, Gwyther SG. New guidelines to evaluate the response to treatment in solid tumors. European Organization for Research and Treatment of Cancer, National Cancer Institute of the United States, National Cancer Institute of Canada. J Natl Cancer Inst. 2000; 92:205-16.

30. Champiat S, Dercle L, Ammari S, Massard C, Hollebecque A, Postel-Vinay S, Chaput N, Eggermont A, Marabelle A, Soria JC, Ferté C. Hyperprogressive Disease Is a New Pattern of Progression in Cancer Patients Treated by Anti-PD-1/PD-L1. Clin Cancer Res Off J Am Assoc Cancer Res. 2017; 23:1920-8. https://doi.org/10.1158/1078-0432. CCR-16-1741.

31. WHO handbook for reporting results of cancer treatment. Geneva (Switzerland): World Health Organization Offset Publication No. 48. 1979.

32. Miller AB, Hoogstraten B, Staquet M, Winkler A. Reporting results of cancer treatment. Cancer. 1981; 47:207-14.

33. Eisenhauer EA, Therasse P, Bogaerts J, Schwartz LH, Sargent D, Ford R, Dancey J, Arbuck S, Gwyther S, Mooney M, Rubinstein L, Shankar L, Dodd L, et al. New response evaluation criteria in solid tumours: revised RECIST guideline (version 1.1). Eur J Cancer Oxf Engl 1990. 2009; 45:228-47. https://doi.org/10.1016/j.ejca.2008.10.026.

34. Ribas A, Chmielowski B, Glaspy JA. Do we need a different set of response assessment criteria for tumor immunotherapy? Clin Cancer Res Off J Am Assoc Cancer 
Res. 2009; 15:7116-8. https://doi.org/10.1158/1078-0432. CCR-09-2376.

35. Chiou VL, Burotto M. Pseudoprogression and ImmuneRelated Response in Solid Tumors. J Clin Oncol Off J Am Soc Clin Oncol. 2015; 33:3541-3. https://doi.org/10.1200/ JCO.2015.61.6870.

36. Nishino M, Giobbie-Hurder A, Gargano M, Suda M, Ramaiya NH, Hodi FS. Developing a common language for tumor response to immunotherapy: immune-related response criteria using unidimensional measurements. Clin Cancer Res Off J Am Assoc Cancer Res. 2013; 19:3936-43. https://doi.org/10.1158/1078-0432.CCR-13-0895.

37. Bohnsack O, Ludajic K, Hoos A. Adaptation of the immune-related response criteria: irRECIST. Ann Oncol. 2014; 25:iv361-72.

38. Seymour L, Bogaerts J, Perrone A, Ford R, Schwartz LH, Mandrekar S, Lin NU, Litière S, Dancey J, Chen
A, Hodi FS, Therasse P, Hoekstra OS, et al. iRECIST: guidelines for response criteria for use in trials testing immunotherapeutics. Lancet Oncol. 2017; 18:e143-52. https://doi.org/10.1016/S1470-2045(17)30074-8.

39. Bennett CL, Silver SM, Djulbegovic B, Samaras AT, Blau CA, Gleason KJ, Barnato SE, Elverman KM, Courtney DM, McKoy JM, Edwards BJ, Tigue CC, Raisch DW, et al. Venous thromboembolism and mortality associated with recombinant erythropoietin and darbepoetin administration for the treatment of cancer-associated anemia. JAMA. 2008; 299:914-24. https://doi.org/10.1001/jama.299.8.914.

40. Larkin J, Chiarion-Sileni V, Gonzalez R, Grob JJ, Cowey CL, Lao CD, Schadendorf D, Dummer R, Smylie M, Rutkowski P, Ferrucci PF, Hill A, Wagstaff J, et al. Combined Nivolumab and Ipilimumab or Monotherapy in Untreated Melanoma. N Engl J Med. 2015; 373:23-34. https://doi.org/10.1056/ NEJMoa1504030. 